\title{
Validation of body fat assessment with a portable hand-to-hand bio-electric impedance system with air displacement plethysmography and hand-to-foot bio-electric impedance system, in a multi-ethnic population aged between 18-55 years
}

\author{
N. Daskalopoulos and D. Bhakta \\ Public Health Nutrition Research Group, Faculty of Life Sciences \& Computing, London Metropolitan University, \\ London $N 78 D B$
}

Body fat is strongly associated with the risk of several chronic diseases such as hypertension, dyslipidemia, Type 2 diabetes and coronary heart disease ${ }^{(1)}$. The portable hand-to-hand bio-electric impedance system (HH BIS, Lloyds Pharmacy Hand Held Body Fat Monitor) is a simple, quick, non-invasive and cost-effective method for assessing body composition. We conducted a validation study to evaluate its usefulness in large epidemiological studies by comparing the portable hand-held system with hand-to-foot bioelectric impedance system (HF BIS, Tanita ${ }^{\circledR}$ MC-980, Multi Frequency Segmental Body Composition Monitor) and with the reference measure of air displacement plethysmography (ADP, BOD POD ${ }^{\circledR}$, Body Composition Tracking Monitors) in a multi-ethnic population. The priori hypothesis was that HH BIS would perform within acceptable limits of agreement $( \pm 1.96 \mathrm{SD})$ in comparison with ADP and HF BIS ${ }^{(2)}$.

An opportunistic sample of the multi-ethnic healthy student population aged between 18-55 years from London Metropolitan University $(n 100)$ were recruited and the mean of two measurements were recorded for each of the three methods. All measurements were conducted by the same individual following a standardised protocol. The mean per cent body fat using the HH BIS (22.4\% 5.9 SD) was similar with ADP (22.7\% 8.4 SD) but higher than measurements obtained with the HF BIS (19.4\% 7.5 SD). Spearman correlations between HH BIS and ADP showed significant association $(r 0.78 P<0.001)$ as with HF BIS $(r 0.80 P<0.001)$ although greater association was observed between HF BIS and ADP $(r \quad 0.86 P<0.001)$. The limits of agreement for HH BIS and ADP (Fig.1) and HF BIS (Fig. 2) were within acceptable limits and comparable to HF BIS and ADP (Fig. 3) for the whole population.

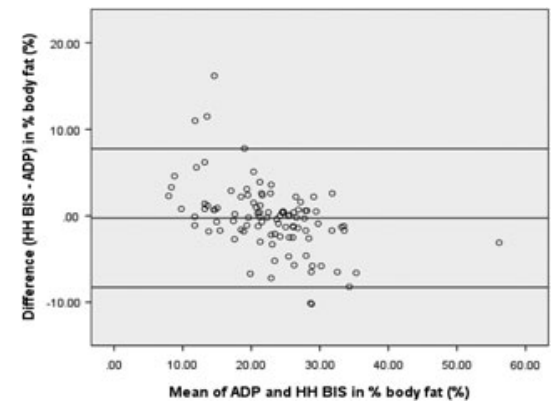

Fig. 1. Hand-to-hand BIS and ADP

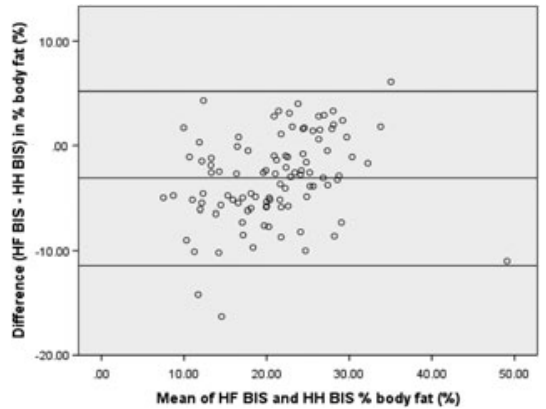

Fig. 2. Hand-to-hand BIS and Hand-to-foot

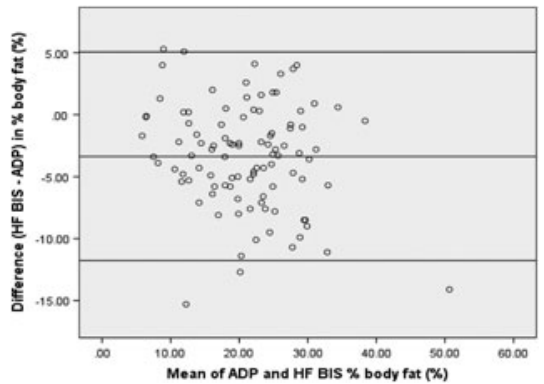

Fig. 3. Head-to-foot BIS and ADP BIS

Significant correlations were observed between the HH BIS and ADP when participants were grouped by gender; male $(n$ 73) and female ( $n$ 27) participants $(r 0.64 P<0.001, r 0.86 P<0.001$, respectively) and level of adiposity; healthy BMI $18-24.9$ ( $n$ 49) and BMI $>24.9$ ( $n$ 51) $(r 0.70 P<0.001, r 0.77 P<0.001$, respectively). However, correlations obtained between the two methods when participants were grouped by ethnicity (White $n$ 60, Black $n$ 27, Asian $n 13)$ were less comparable $(r 0 \cdot 86 P<0 \cdot 001, r 0 \cdot 68 P<0 \cdot 001, r 0 \cdot 29$ $N S$, respectively).

We observed acceptable agreement between hand-to-hand BIS and air displacement plethysmography for large scale populations, but would heed caution for its use for ethnic groups.

1. Dehghan M, Merchant AT (2008) Nutr J 7, 26-32.

2. Ward LC (2013) E J Clin Nutr 67, S10-S13. 